\title{
Study on Dust Turbulence-Chemical Agglomeration for Electrostatic Precipitation Technology
}

\author{
Gao Shan ${ }^{1, a}$, XIAO Li-chun ${ }^{1, b^{*}}$ \\ ${ }^{1}$ College of environmental and chemical engineering, Yanshan University, Qinhuangdao, Hebei ,China
}

\begin{abstract}
Due to the turbulent mixing between agglomeration agent and dust, the number of collisions per unit time between particles and droplets increases with the particle size in the process of chemical agglomeration, and the agglomeration efficiency increases accordingly. Turbulent agglomeration can promote the agglomeration effect of subsequent chemical agglomeration by strengthening the collision between particles and agglomerant droplets. The author used chemical agglomeration and turbulent mixing to cooperate in order to improve the efficiency of dust removal. Turbulent mixing can promote chemical agglomeration from agglomeration effect and dust removal efficiency, which can greatly improve electric dust removal technologyTurbulent mixing is the most intense at the inlet box position, and the agglomeration effect is the best. Turbulent mixing synergistic effect has an effect on dust removal efficiency. Compared with the three curves, it can be seen that the dust removal efficiency increases rapidly with the increase of agglomeration concentration, the curve trend changes obviously. The dust removal efficiency can reach $98.36 \%$ and it is the highest in the middle section when the wind speed is greater than $11.2 \mathrm{~m} / \mathrm{s}$. Through the experiment, the turbulent mixing and chemical coagulation method has a good application prospect in the electrostatic precipitators.
\end{abstract}

\section{Introduction}

In recent years, the atmospheric environment continues to deteriorate, haze weather occurs frequently, which seriously endangers human health and restricts the sustainable development of economy. Coal plays an important role in the energy structure of our country, in which coal-fired power generation is the main way of coal consumption and brings serious damage to the atmospheric environment

In order to alleviate this situation, it is necessary to develop new coal power generation technology, improve the conversion rate and utilization rate of resources, and reduce the collection and disposal of soot, harmful gases and wastewater in the process of electric power production. Coal gasification combined cycle (IGCC) power generation technology is considered to be one of the most promising clean coal power generation technologies, which has obvious advantages in improving power generation economy and reducing pollutant emission ${ }^{[1]}$. When the crude gas is used for IGCC power generation, in order to realize the safe and reliable operation of IGCC and clean power generation, the impurity must be removed before the crude gas enters the power generation equipment. Although the dust removal efficiency of gasifier gas is higher than that of ordinary dust removal device, it is difficult to capture the fine particle dust in gasifier gas because it is relatively large, which affects the effect of dust removal. These fine

a1195283047@qq.com

* Corresponding author: bxlc@ysu.edu.cn particles will enrich a large number of corrosive substances, have an impact on the safe operation of generator sets, and finally discharge into the atmosphere, which is more harmful to human health, so it is of great significance to study the removal methods of fine particles.

The dust particle size of gasifier is small and the following property of air flow is better. When turbulence disturbance occurs in the flow field, the motion state of dust becomes chaotic and disordered, which increases the collision probability with other particles, and the agglomeration efficiency increases ${ }^{[2-3]}$. When the atomization droplets are agglomerated, the surface polarity of the atomized droplets is strong, and the fine particles in the flue gas will adhere to the surface of the atomized droplet as soon as they contact the atomized droplet $^{[4-5]}$, and any contact between the particles and the droplet will attachment deposition. The collision probability between particles and droplets becomes an important factor affecting agglomeration efficiency ${ }^{[6-7]}$. Turbulent agglomeration mainly depends on the turbulent entrainment of the fluid in the turbulent flow field ${ }^{[8-9]}$. The particles with different inertia produce velocity difference in the disturbed flow field, which leads to the increase of collision probability between each other and improves the agglomeration efficiency. Turbulent agglomeration technology can promote the agglomeration effect of subsequent chemical flow agglomeration by increasing the probability of particle 
collision $^{[10-11]}$. Therefore, in order to further improve the dust removal effect, the turbulence and chemical agglomeration of gasifier dust in electrostatic precipitation technology are studied ${ }^{[12]}$.

\section{Experimental Methods and Devices}

\subsection{Experimental Methods}

Under certain conditions, the agglomeration liquid is sprayed into the pipe at different positions to agglomerate with the dust, so that the dust-containing airflow enters the electrostatic precipitator to purify under the action of electric field, collect the collected dust, and measure its particle size distribution.

\subsubsection{Measurement of agglomeration effects}

Under certain conditions, the agglomeration liquid is injected into the pipe to agglomerate with the dust, and the dust is removed by the electrostatic precipitator under the action of air flow. After the experiment, the dust is collected and removed. The particle size distribution of dust was measured by particle size distribution instrument, the agglomeration process was analyzed, and the agglomeration effect of different agglomerates was observed.

\subsubsection{Measurement of dust concentration}

The sampling device consists of a wet gas flow meter, a buffer bottle, an air pump, a drying bottle, and a sampling tube, as shown in Fig 1 Isokinetic sampling method is used to measure the concentration of dust inlet and outlet. After connecting the device according to the diagram, the sampling gun is placed in the designated position, and the measurement begins. After sampling, the dust concentration and dust removal efficiency of the dust collector are calculated according to the quality difference of the weighing filter tube mentioned above.

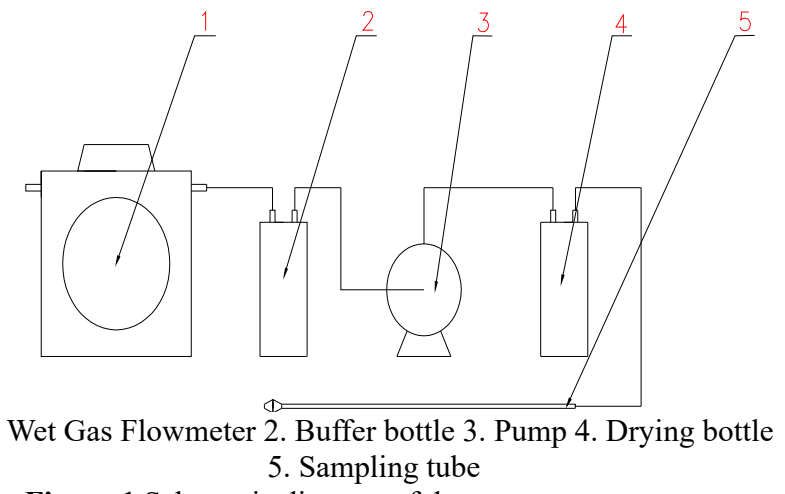

Figure 1 Schematic diagram of dust content test system

\subsection{Experimental Devices}

This test adopts the electrostatic precipitator, which mainly consists of 6 parts, which are dust feed system, low voltage control power supply system, high voltage control power supply system, fan system, discharge dust collection system and spray system. The experimental device is shown in Fig 2. When the wet dust collector is running, firstly turn on the main control switch, turn on the low voltage control system, then adjust the required wind speed and water pressure, turn on the high voltage power supply system after the adjustment, Finallytadjust the required high voltage power supply.

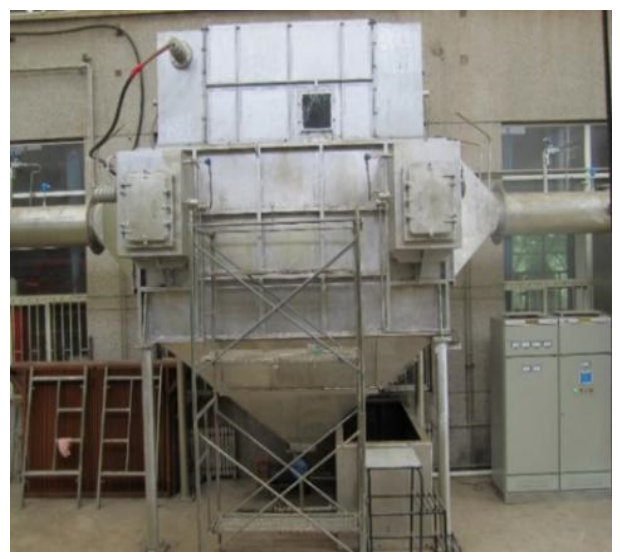

Figure 2 Schematic diagram of the ESP system

\section{Results \& Discussion}

\subsection{Study on the Effect of Turbulent Mixing Synergy on Agglomeration Performance}

Spraying agglomerates in front of the main body of the dust collector can promote the agglomeration effect of dust. Adding surfactants can further improve the agglomeration effect, but at the same time, the use of other chemical reagents will also bring new pollution and economic cost problems. Considering that the agglomeration agent is sprayed into the dust generator in the above experiment, it collides with the dust quickly after entering the pipe, and the air disturbance between them is small. In order to find a more economical and environmentally friendly agglomeration method, the effect of turbulent mixing caused by air disturbance between dust and agglomeration on dust agglomeration effect was further explored by changing the injection position of agglomeration agent. The following experiments were carried out.

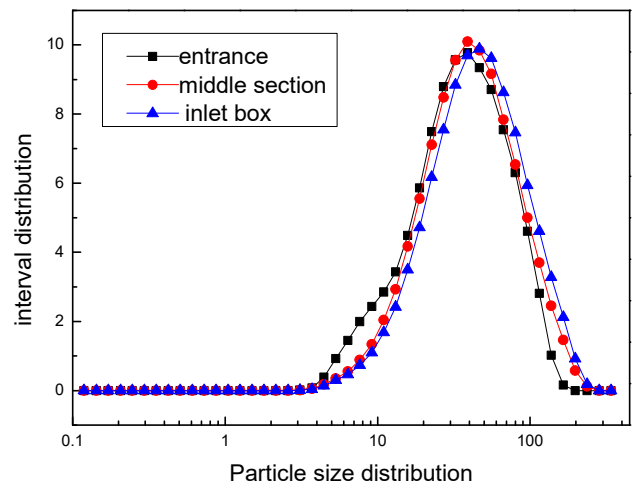

Figure 3 Distribution of Turbulent Mixing on Dust Particle Size 
As shown in Fig 3, it can be seen that the inlet box and the middle section curve shift to the right than the inlet curve, the inlet box curve shift to the greater right; the particle size is between $0 \mu \mathrm{m}-10 \mu \mathrm{m}$, the entrance content is obviously higher than the middle section and the inlet box, the middle section is slightly higher than the inlet box, the diameter is between $10 \mu \mathrm{m} \sim 100 \mu \mathrm{m}$, the inlet box curve is close to the middle section and the entrance curve. The peak value of the three curves is in turn is the inlet box $>$ the middle section $>$ the entrance.

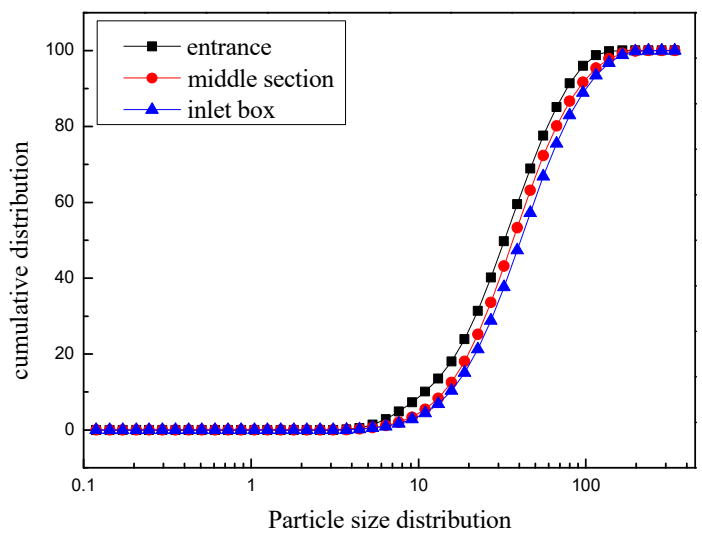

Figure 4 Cumulative distribution of turbulent mixing on dust agglomeration

As shown in Fig 4, it can be seen that the inlet box and the middle section curve shift to the right than the inlet curve, the inlet box curve shift to the greater right; the particle size is between $0 \mu \mathrm{m} \sim 10 \mu \mathrm{m}$, the entrance content is obviously higher than the middle section and the inlet box, the middle section is slightly higher than the inlet box, the diameter is between $10 \mu \mathrm{m}-100 \mu \mathrm{m}$, the inlet box curve is close to the middle section and the entrance curve. The peak value of the three curves is in turn the inlet box $>$ the middle section $>$ the entrance. Analysis reason: there is a velocity difference between the agglomerated liquid in the middle section and the dust in the pipeline, which is trapped by droplets in the flue. However, there is no obvious relative motion between droplets and, resulting in the mixed collision is not strong and the turbulent mixing is not obvious. However, due to the uneven distribution of airflow velocity and strong air disturbance, turbulence mixing is more intense and agglomeration effect is better.

\subsection{Effect of turbulence mixing on dust removal efficiency}

\subsubsection{Effect of turbulence mixing on dust removal efficiency}

Table 1 Effect of turbulent mixing on dust removal efficiency

\begin{tabular}{lccc}
\hline $\begin{array}{c}\text { Agglomerate } \\
\text { spray position }\end{array}$ & Entrance & $\begin{array}{c}\text { Middle } \\
\text { section }\end{array}$ & Inlet box \\
\hline $\begin{array}{c}\text { Dust removal } \\
\text { efficiency(\%) }\end{array}$ & $97.72 \%$ & $97.83 \%$ & $98.03 \%$ \\
\hline
\end{tabular}

As shown in table 1, it can be seen that the dust removal efficiency of the three locations $>$ the inlet $>$ the middle section of the intake box, and the dust removal efficiency of the three is $98.03 \%$ respectively. The results show that the dust removal efficiency is affected by spraying chemical aggregate at different positions. This is consistent with the effect of turbulent mixing on dust agglomeration in the above experiments. The reason of this phenomenon is that the fine particles are in contact with the chemical agglomeration at the entrance when they fall from the dust generator, so there is no turbulent mixing between them, there is only chemical agglomeration, the agglomeration effect is general, and the dust removal efficiency is the lowest; The dust removal efficiency is higher than that at the entrance, but the dust removal efficiency is not obvious because the injection position is closer to the main body of the dust collector and the agglomeration time is shortened. Compared with the middle section, the dust removal efficiency of the intake box is higher because the wind speed of the pipeline is obviously larger than that of the middle section, the turbulent mixing flow is more intense, the dust agglomeration effect is better, and the particle size is larger. So dust removal efficiency is higher.

\subsubsection{Effect of concentration of agglomerates on dust removal efficiency}

Based on past experience, XTG agglomerates were selected to change the concentration of chemical aggregates at the entrance, middle section and inlet box respectively, and the effect of concentration on dust removal efficiency under turbulent mixing are studied.

The experimental results are shown in Fig 5. We can see that the dust removal efficiency increases with the increase of agglomeration concentration at the three locations, and the agglomeration concentration increases obviously between $0.02 \mathrm{~g} / \mathrm{L}$ and $0.10 \mathrm{~g} / \mathrm{L}$, and does not change between $0.10 \mathrm{~g} / \mathrm{L}$ and 0.15 . Compared with the changing trend of the three curves, it can be seen that the dust removal efficiency increases rapidly with the increase of agglomeration liquid concentration, the curve trend changes obviously, the highest dust removal efficiency can reach $98.36 \%$, and the middle section curve also changes with the increase of agglomeration liquid concentration. Analysis of the reasons for this phenomenon: the concentration of agglomeration solution has a certain effect on turbulent mixing, the greater the concentration of agglomeration solution, the more obvious the effect of turbulent mixing, increase the probability of collision between dust and agglomeration agent, promote the combination of dust and agglomeration effect. Considering the cost saving from the economic point of view, selecting the appropriate concentration of agglomeration liquid is not only conducive to production efficiency, but also conducive to environmental protection and practical benefits. 


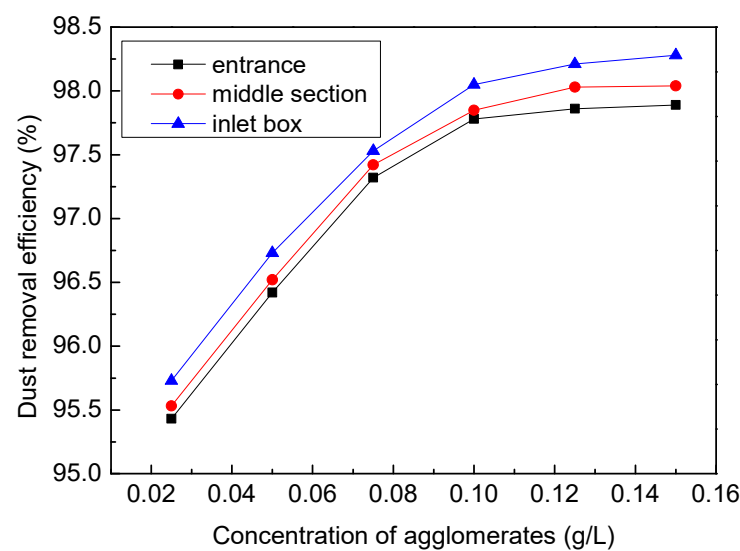

Figure 5 Dust removal efficiency diagram under different concentration of agglomerates

\subsubsection{Effect of flue gas velocity on dust removal efficiency}

At certain experimental conditions, the chemical aggregate was injected into the middle section and the wind speed of the pipeline was changed to $6.7 \mathrm{~m} / \mathrm{s} 、 9 \mathrm{~m} / \mathrm{s}$, $11.2 \mathrm{~m} / \mathrm{s} 、 13.4 \mathrm{~m} / \mathrm{s} 、 15.7 \mathrm{~m} / \mathrm{s}$ respectively to explore the effect of wind speed on turbulent mixing and the effect on dust removal efficiency.

As shown in Fig. 6, it can be seen that the dust removal efficiency increases with the increase of pipeline wind speed at the middle section, the change trend is obvious between $6.7 \mathrm{~m} / \mathrm{s} \sim 11.2 \mathrm{~m} / \mathrm{s}$ of pipeline wind speed, and the curve trend tends to be stable after wind speed is greater than $11.2 \mathrm{~m} / \mathrm{s}$. When the wind speed is $11.2 \mathrm{~m} / \mathrm{s}$, the dust removal efficiency is $97.98 \%$, and when the wind speed is $15.7 \mathrm{~m} / \mathrm{s}$, the dust removal efficiency is $98.32 \%$.

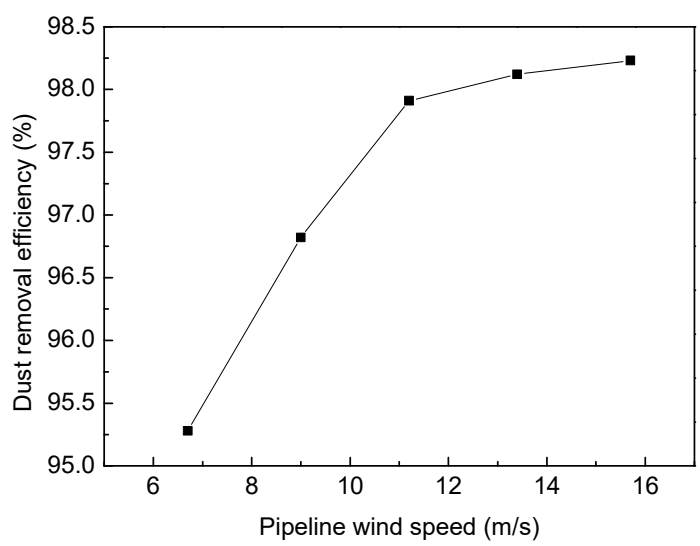

Figure 6 Dust removal efficiency diagram under different wind speed conditions

The reasons for this are as follows: with the increase of flue gas velocity in turbulent concentrator, the turbulence intensity, turbulent kinetic energy and turbulent dissipation rate increase. The turbulent agglomeration kernel function increases and the collision and agglomeration between fine particles and droplets are promoted, thus the agglomeration efficiency and particle size increase. The increase of flue gas velocity is beneficial to increase turbulence disturbance and promote agglomeration effect, but the increase of velocity is limited in practical application considering energy consumption and other reasons. As the flue gas velocity continues to increase, the excessive flue gas velocity will affect the residence time of particulate matter in the turbulent agglomeration, which will lead to the decrease of particle agglomeration efficiency. In addition, at higher flue gas velocity, the relative velocity increases due to the mass and inertia of different particle sizes, and when the relative velocity between particles is greater than its critical relative velocity, the remaining energy after collision is greater than van der Waals force between particle surfaces, and the collision between particles does not lead to agglomeration. Therefore, on the one hand, the excessive flue gas velocity reduces the residence time of fine particles in turbulent flow field, on the other hand, it will lead to invalid collision between some particles, and even reduce the agglomeration efficiency between fine particles.

\section{Conclusions}

The improvement of chemical agglomeration effect mainly depends on the preparation of cheaper and more efficient chemical agglomeration agents. Therefore, under the condition of limited performance of chemical agglomerates, it is difficult to further improve the agglomeration effect, and the extensive use of chemical agglomerates will cause additional pollution to the environment. Turbulent mixing can promote chemical agglomeration from agglomeration effect and dust removal efficiency, which can greatly improve electrostatic precipitation technology. Turbulent mixing is the most intense at the inlet box position, and the agglomeration effect is the best; Turbulent mixing synergistic effect has an effect on dust removal efficiency. Compared with the three curves, it can be seen that the dust removal efficiency increases rapidly with the increase of agglomeration concentration, the curve trend changes obviously, the dust removal efficiency can reach $98.36 \%$ and the dust removal efficiency is the highest in the middle section the wind speed is greater than $11.2 \mathrm{~m} / \mathrm{s}$.

\section{Acknowledgments}

This research was supported by the Natural Science Foundation of Hebei province, China (E2015203236). It was also supported by the Science and Technology Plan of Qinhuangdao (202003B033).

\section{References}

1. Sun Xudong, Zhang Bo, Peng Suping. Development Trend and Strategic Countermeasures of 2035 Clean Coal Technology in China [J].] Study China Engineering Science,2020,22(03):132-140.

2. Bid, Sun Zongkang, Zhou Lei, Hu Bin, Yuan Zhulin, Yang Linjun. Experimental Study on the Coupling of Turbulence and Chemical Agglomeration to Promote the Removal of Coal-fired Particles by Electric Dust [J]. Journal of Electrical Engineering of 
China,2019,39(10):2954-2962.

3. Zheng Jianxiang, Xu Shuai, Wang Jingyang. A Study on Superfine Particle Aggregation Model and Turbulent Converter Aggregation [J].]; and Journal of Electrical Engineering of China,2016,36(16):4389-4395+4524.

4. Liu Jiaxun. Experimental study on chemical agglomeration of fly ash from coal combustion [D].] and its mechanism analysis Harbin University of Technology ,2008.

5. Sun Zongkang,Yang Linjun,Wu Hao,Wu Xin. Effect of turbulent flow field properties on improving the removal of coal-fired fine particles in chemical-turbulent agglomeration[J]. Advanced Powder Technology,2020,31(1).

6. Torben Schæfer. Growth mechanisms in melt agglomeration in high shear mixers[J]. Elsevier B.V.,2001,117(1).

7. Li Hailong, Zhang barracks, Zhao Yongchun, Zhang Kai, Zhang Liqi, Zheng Chuguang. Study on Physicochemical Properties and Wetting Mechanism of Fly Ash J]. Coal-fired Coal Journal of Engineering Thermophysics ,2009,30(09):1597-1600.

8. Rajniak P,Mancinelli C,Chern R T,Stepanek F,Farber L,Hill B T. Experimental study of wet granulation in fluidized bed: impact of the binder properties on the granule morphology.[J]. International journal of pharmaceutics,2007,334(1-2).

9. Elizaveta Forbes. Shear, selective and temperature responsive flocculation: A comparison of fine particle flotation techniques[J]. International Journal of Mineral Processing,2011,99(1).

10. Boris V. Balakin,Kirill V. Kutsenko,Alexey A. Lavrukhin,Pawel Kosinski. The collision efficiency of liquid bridge agglomeration[J]. Chemical Engineering Science,2015,137.

11. Wei Feng,Zhang Jun-ying,Zheng Chu-guang. Agglomeration rate and action forces between atomized particles of agglomerator and inhaled-particles from coal combustion.[J]. Journal of environmental sciences (China),2005,17(2).

12. Wang Yuxiang. Design and numerical simulation of fine particle chemical agglomeration system in 300MW coal-fired power station [D].]; and Huazhong University of Science and Technology ,2011. 